\title{
Calcaneal Myxoid Chondrosarcoma: A Rare Case
}

\author{
Joshi $A,{ }^{1}$ Shahi $R,{ }^{2}$ Singh $S,{ }^{3}$ Chand $P,{ }^{1} K C B R^{1}$ \\ 'Department of orthopedics, 'Department of radiology, ${ }^{3}$ Department of pathology, Shree Birendra Hospital, Chhauni, \\ Kathmandu, Nepal.
}

\begin{abstract}
Skeletal myxoid chondrosarcoma is an extraordinarily rare neoplasm with a distinct histological morphology. Although it grows slowly, the clinical course was found to be worse than usual chondrosarcoma. We report a case of a Skeletal myxoid chondrosarcoma in the calcaneus of a 21-year-old female who presented to us with a feature of gradually increasing benign swelling left foot for last two years. Initial investigations were indicating towards a benign lesion for which excision of the lesion was performed. But intra operative findings were suggestive of an aggressive lesion, and to our utter surprise histopathologically it turned out to be Myxoid Chondrosarcoma. Because of its high malignant nature below knee amputation was performed as second definitive surgery. Since her clinical course and radiological pictures were suggestive of benign lesion we got carried away with a diagnosis of chondrmyxoid fibroma and excision of the lesion was attempted without attempting histopathological diagnosis by less invasive methods.
\end{abstract}

Keywords: caldaneal; chondrosarcoma; myxoid.

\section{INTRODUCTION}

Skeletal myxoid chondrosarcoma is extremely rare neoplasm with very few cases published with scanty references. Calcaneal myxoid chondrosarcoma is even rarer as SMC are commonly reported to occur in femur. ${ }^{1}$ The aim of this article is to report myxoid chondrosarcoma in an unusual location (calcaneus), which was initially diagnosed as a benign lesion. We want to emphasize on the fact that many bone neoplasm mimic a benign condition initially, a thorough investigation and a histopathological diagnosis before instituting a surgical procedure is important to avoid multiple surgeries for same condition.

\section{CASE REPORT}

A 21 year old female presented to us with a two years history of gradually increasing swelling on right heel associated with intermittent pain aggravated by activities. For last three months, the pain became intense with difficulty in walking. There was a non inflammatory, non pulsatile, non compressible swelling measuring $7 \times 3 \mathrm{Cm}$ (Figure 1). The mass was cystic with well defined margins, adherent to underlying structures but overlying skin was free. The tinel's sign was positive. On the lateral view of ankle radiograph, there was a well defined osteolytic lesion in the calcaneous (Figure 2). The lesion was lobulated and demarcated by a thin margin of sclerosis with narrow

Correspondence: Dr. Amit Joshi, Department of orthopedics, Shree Birendra Hospital, Chhauni, Kathmandu, Nepal. Email: dramitjoshi7@ gmail.com. 
zone of transition. On MRI the lesion was a lobulated well defined mass which was hypointense in T1 and bright in T2 image (Figure 3). Considering the X-ray and MR imaging findings, which suggested a benign tumor with a myxoid component and fibrous septae, the initial radiological diagnosis was chondromyxoid fibroma, or intraosseous lipoma. Excision of the tumor and currettage was done, intraoperatively the leson was myxomatous and was invading the calcaneum creating multiple loculi in the calcaneum. The loculi were broken and cavity was packed with cancellous bone graft. Post operative period was uneventful but to our utter surprise the HPE report came out to be Myxoid chondrosarcoma. After having detail discussion with the pathologist a final diagnosis of SMC was made based upon the available evidences as patient refused for immunohistochemistry. Further CT Scan of chest was done to rule out distant metastasis after which patient was counseled for below knee amputation.
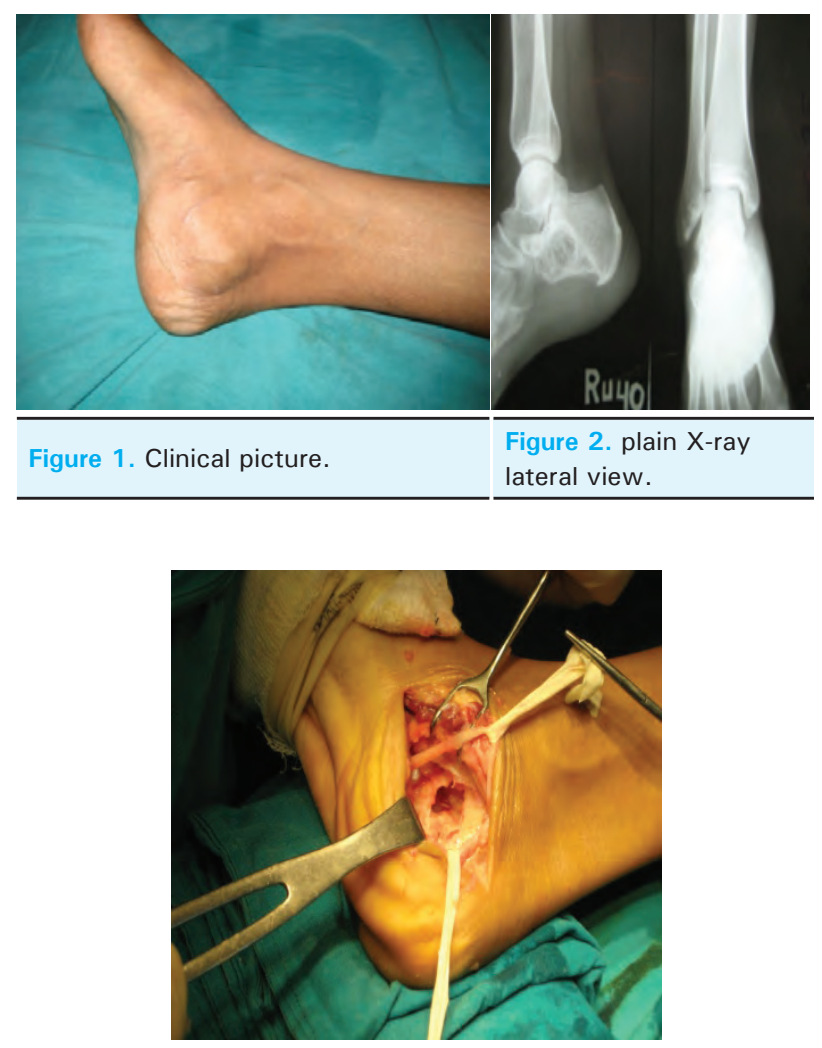

Figure 3. intraoperative picture showing erosion of the calcaneum.

\section{DISCUSSION}

In view of the rare incidence of SMC and that to in an unusual location like calcaneum makes our case more interesting. In a recent case report of Jong WK et al. they claimed that their case of myxoid chondrosarcoma in the calcaneus was third ever reported. ${ }^{2}$ So to best of our knowledge our case report will be the fourth one ever reported. SMC has not been fully evaluated as distinct clinicopathological entity, and the term is used interchangeably to designate either a conventional chondrosarcoma with prominent myxoid degeneration or a myxoid sarcoma that is identical histologically to extraskeletal myxoid chondrosarcoma (EMC). This may be the reason for very few case reports in the literature. Considerable controversy surrounds the question of whether EMC and chordoid sarcoma are the same entity or separate ones. ${ }^{3}$ Although similar light microscopic features are noted in EMC and SMC, fundamental differences are noted at the ultra structural and molecular levels, suggesting that EMC and SMC represent two distinct entities in the chondrosarcoma family of tumors. ${ }^{4}$

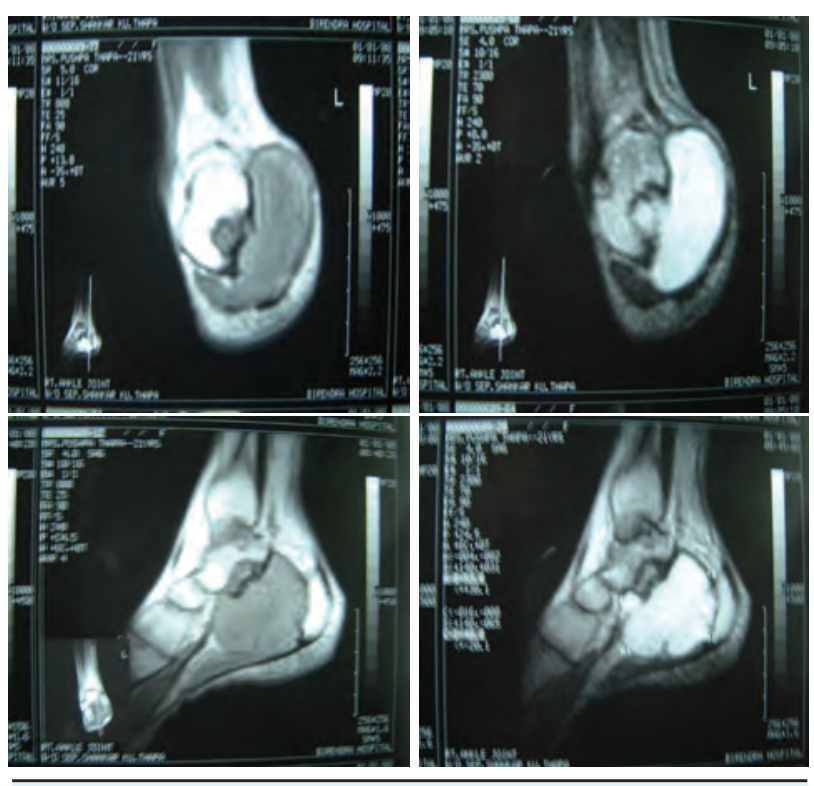

Figure 4. MRI Pictures of the lesion.

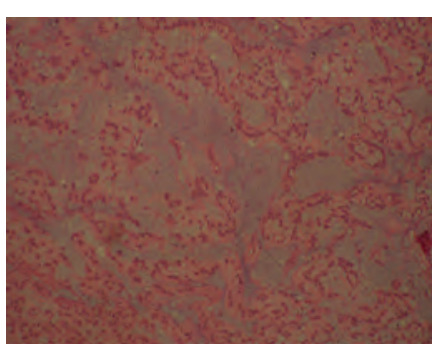

Figure 5A. interconnected cords, strands and loose clusters of eosinophilic spindle shaped tumor cells separated by abundant myxoid stroma.

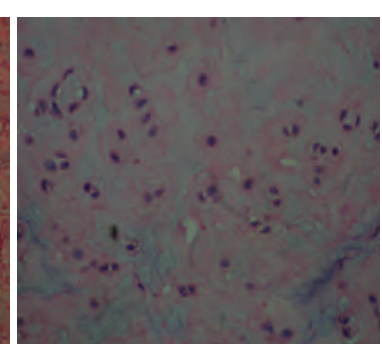

Figure 5B. iRim of eosinophilic cytoplasm surrounding loosly clustered tumor cells with hyperchromatic nuclei floating in myxoid matrix.
A benign looking clinical course and radiological picture as in our case was mentioned by Jong WK, where as others have described the aggressive radiological and 
clinical courses. ${ }^{1,2,5}$

Preoperative radiologic diagnosis of our case was chondromyxoid fibroma based on the X-ray and MR imaging findings, which suggested a benign lesion with a myxoid component and fibro septation. Also, the young age of the patient led to a more benign impression of the lesion. It is cited that skeletal myxoid chondrosarcoma is distinguished from chondromyxoid fibroma mainly by the sharply demarcated radiographic area of bone destruction. ${ }^{5}$ However, our case showed no area of bone destruction, making the differential diagnosis difficult. Because of all these reasons we directly planned for excisional biopsy neglecting less invasive diagnostic techniques. Only during surgery we could appreciate its non benign nature because of its invasion and destruction of the calcaneum. Since we are not having facilities of frozen section we had to wait till the HPE report for final diagnosis. Although her CT scan of chest showed no metastatic lesion, the calcaneal lesion had a large soft tissue component indicating extra compartmentalization of tumor and high histological grade led to stage the tumor as IIB (Ennekin's) for which radical surgery (Above Knee Amputation) was performed.

One controversy still remained regarding its skeletal or extraskeletal origin. In some references, skeletal and extraskeletal myxoid chondrosarcoma are described to be morphologically identical, and imaging studies are necessary to confirm the bone or soft tissue origin of the tumor. ${ }^{6}$

In our case since the soft tissue component was larger than the bony we debated that it is of soft tissue origin but pathologist insisted it to be as skeletal SMC because of the characteristic histological features.

In another study, skeletal myxoid chondrosarcoma is said to be morphologically distinct from the extraskeletal counterpart by virtue of the lobulated, multinodular appearance, comprised of a uniform population of rounded to slightly spindled cells. The neoplastic cells with hyperchromatic nuclei and inconspicuous nucleoli are described to be arranged in anastomosing chords, strands, and nests and pseudopapillary structures embedded in an abundant, mostly hypovascular, mucinous matrix. ${ }^{7}$ Because of similar histological picture in our case we came to a final diagnosis of SMC (Figure 5 A,B). Although S-100 protein has been mentioned to be specific for SMC, cases of myxoid chondrosarcoma lacking S-100 immunopositivity have been reported in the literature. ${ }^{8}$ Patient couldn't perform immunohistochemistry because of economical constrains.

Many bone neoplasm mimic a benign condition initially, even if it looks widely excisable, a thorough investigation and a histopathological diagnosis before instituting a surgical procedure is important to avoid multiple surgeries for same condition.

\section{REFERENCES}

1. Antonescu CR, Argani P, Erlandson RA, Healey JH, Ladanyi M, Huvos AG. Skeletal and extraskeletal myxoid chondrosarcoma: a comparative clinicopathologic, ultrastructural, and molecular study. Cancer. 1998;83:1504-21.

2. Jong WK, Jung AC, Kyu-Sung K, Joo HO, Jin HC, Heung SK. Myxoid chondrosarcoma in the calcaneus: a case report with MR imaging findings. Skeletal Radiol. 2007;36:S82-S85.

3. Dardick I, Lagace R, Carlier MT, Jung RC. Chordoid sarcoma (extraskeletal myxoid chondrosarcoma): a light and electron microscopic study. Virchows Arch A Pathol Anat Histopathol. 1983;399:61-78.

4. Cristina RA, Pedram A, Robert AE, John HH, Marc L, Andrew GH. Skeletal and Extraskeletal Myxoid Chondrosarcoma: A Comparative Clinicopathologic, Ultrastructural, and Molecular Study. 1998;83(8):1504-21.
5. Steiner G, Greenspan A, Jahss M, Norman A. Myxoid chondrosarcoma of the os calcis: a case report. Foot Ankle. 1984;5:84-91.

6. Weiss SW, Coldblum JR. Cartilaginous soft tissue tumors. In: Enzinger and Weiss's soft tissue tumors. London: Mosby; 2001.

7. Kilpatrick SE, Inwards CY, Fletcher CD, Smith MA, Gitelis S. Myxoid chondrosarcoma (chordoid sarcoma) of bone: a report of two cases and review of the literature. Cancer. 1997;79:1903-10.

8. Abramovici LC, Steiner GC, Bonar F.Myxoid chondrosarcoma of soft tissue and bone: a retrospective study of 11 cases. Hum Pathol. 1995;26:1215-20. 